\title{
Irreversibility and Entanglement Spectrum Statistics in Quantum Circuits
}

\author{
Daniel Shaffer ${ }^{1}$, Claudio Chamon ${ }^{1}$, Alioscia Hamma ${ }^{2}$ and \\ Eduardo R. Mucciolo ${ }^{3}$ \\ ${ }^{1}$ Department of Physics, Boston University, Boston, Massachusetts 02215, USA \\ ${ }^{2}$ Center for Quantum Information, Institute for Interdisciplinary Information \\ Sciences, Tsinghua University, Beijing 100084, P.R. China \\ ${ }^{3}$ Department of Physics, University of Central Florida, Orlando, Florida 32816, USA
}

\begin{abstract}
We show that in a quantum system evolving unitarily under a stochastic quantum circuit the notions of irreversibility, universality of computation, and entanglement are closely related. As the state evolves from an initial product state, it gets asymptotically maximally entangled. We define irreversibility as the failure of searching for a disentangling circuit using a Metropolis-like algorithm. We show that irreversibility corresponds to Wigner-Dyson statistics in the level spacing of the entanglement eigenvalues, and that this is obtained from a quantum circuit made from a set of universal gates for quantum computation. If, on the other hand, the system is evolved with a non-universal set of gates, the statistics of the entanglement level spacing deviates from Wigner-Dyson and the disentangling algorithm succeeds. These results open a new way to characterize irreversibility in quantum systems.
\end{abstract}

PACS numbers: 89.70.Cf, 03.67.Mn

Keywords: entanglement entropy, chaos, irreversibility, quantum circuits

\section{Introduction}

The onset of irreversibility in physics is one of the great questions at the heart of statistical mechanics. The second principle of thermodynamics in essence states that spontaneous processes happen in one direction, namely increasing entropy or decreasing free energy. Boltzmann's H-theorem was aimed in proving this possibility starting from microscopic time-reversal mechanics, but, as he tragically had to realize, there is no way of doing that. In classical physics irreversibility either has to assume some seed of randomness, or it must resort to coarse graining and topological mixing [1]. Of course, in classical physics, coarse graining and the counting of micro states are arbitrary operations, and it was soon recognized that firmer grounds for statistical mechanics must be found in the quantum domain.

At a first glance, though, the quantum case looks even harder. In a closed system evolution is unitary, and therefore the entropy of a quantum state cannot increase. Moreover, unitary evolution is always reversible, so irreversibility is strictly speaking 
impossible. In classical mechanics irreversibility is due to chaos, that is, high sensitivity to initial conditions. But in quantum mechanics, unitarity implies that slightly different initial conditions do not evolve into highly different states.

Recently, [2], irreversible phenomena like thermalization have been interpreted in terms of entanglement. Because of entanglement, every subsystem of a pure quantum system will typically increase its entropy to give thermal expectation values to most of its observables. However, it is not clear exactly in what way entanglement is involved in the irreversible character of thermalization. In fact, also non thermalizing (integrable) systems increase their entropy to saturation. After one has stated that irreversibility is due to entanglement, we are left with the feeling that one still needs to specify what kind of entanglement. What notion of irreversibility one should use, is part of the question, as well. Moreover, one would like to tackle all these questions in a more general setting than evolution governed by a Hamiltonian. We mentioned above that irreversibility in quantum mechanics cannot happen at the level of wave function. If two initial conditions are slightly different, their overlap in time will remain bounded. This is a direct consequence of unitarity. Instead, one can consider the Loschmidt echo, that is, the overlap between the state obtained by evolving the initial state with some Hamiltonian $H$ and then reversing the evolution with a slightly perturbed Hamiltonian $H+\epsilon V$ : $\mathcal{L}=\left|\left\langle\psi(0)\left|e^{i(H+\epsilon V) t} e^{-i H t}\right| \psi(0)\right\rangle\right|^{2}$. A fast decay of $\mathcal{L}$ is what is called irreversibility [3]. It is remarkable that quantum irreversibility and non-integrability are not as closely connected as one may think [4], which is another sign of the non satisfactory conceptual standing of the whole matter.

In this paper, we take a completely new point of view. We take seriously the idea that the defining feature of quantum mechanics is entanglement. Irreversibility and thermalization must be a consequence of entanglement. As we shall see, it is not the amount of entanglement per se that is important. This is too much of a simple and generic notion. A specific pattern of entanglement, though, is what is important for irreversibility. This approach offers several advantages. First of all, it is novel. Questions that have been asked for decades and remained somehow unresolved can now be looked from a new point of view. In particular, entanglement is not neutral (like energy) with respect to the degrees of freedom that are involved. Entanglement is defined within a chosen tensor product structure, that is, it depends on what the local observables are [5]. Understanding thermalization must mean finding which observables thermalize, i.e, understanding what the relevant tensor product structure is. We posit that if the evolution is irreversible the entanglement will be complex in the degrees of freedom in which the interactions are simple. In this sense irreversibility is a complexity problem. Every Hamiltonian is diagonalizable, but simple interactions may result in complex entanglement. The second advantage is that this approach is apt to study any quantum evolution. One does not need a Hamiltonian, and not even unitary evolution, in principle. Every evolution that takes a quantum density matrix into another quantum density matrix (i.e, a completely positive map), that is, any quantum process, can be studied with this method. 
The method, first introduced in Ref. [6], is easily explained. We evolve the state with a quantum evolution, starting from a completely disentangled state. After a while, the state will be fully entangled. Then we try to go back to the initial state, that is, we try to undo the entanglement. To this end, we use a Metropolis-like algorithm. We select a random local unitary (that is, involving up to two degrees of freedom), and check whether this decreases some measure of entanglement. If yes, we keep it, and we draw another random unitary operator. If not, we reject it. After a certain number of local unitaries, we may have two outcomes: (i) the state is fully disentangled, or, (ii) no matter how long we keep drawing local unitaries, the state remains entangled. In the case (i) we have reversed the evolution trying out a small number of unitaries. Since disentangling the state is easy, we say that entanglement is not complex. On the other hand, the case (ii) is irreversible. We see that irreversibility means the incapability of disentangling the state, unless one tries out all the possible evolutions. Irreversible entanglement is thus complex entanglement.

At this point, we ask ourselves what makes some entanglement complex or not. If the entanglement is complex, one should be able to look at the final state and find a pattern that tells us that there is no way to disentangle it in a simple way. To this end, we look at the level spacing statistics of the reduced density matrix spectrum, namely, the eigenvalues of the reduced density matrix of a subsystem. We show that the irreversible, complex entanglement corresponds to a Wigner-Dyson statistics of the eigenvalue spectral fluctuations, showing a robust level repulsion. On the other hand, simple, removable entanglement possesses spectral fluctuations that follow, for instance, a Poisson statistics, where no level repulsion is present. Therefore, a generic quantum (unitary) evolution is irreversible because of a specific pattern of entanglement. As we explained, irreversibility is thus understood in terms of the complexity of the entanglement, not in terms of how much a state is entangled.

In this paper, we push forward the ideas first proposed in Ref. [6] where this phenomenon was noticed for a specific subset of quantum circuits, namely, those generated by the subgroup of the unitary group realized by the permutation subgroup of unitary transformations. However, the question was raised whether the findings in Ref. 6] are just a property of the particular transformations chosen, or if, instead, the notion of irreversibility based on entanglement makes sense for general quantum unitary evolutions. To this end, we consider a quantum system made of $N$ qubits, and subject to a random unitary quantum circuit. The local unitaries are one- and two-qubit gates. The set containing the gates $\mathbf{H}$ (Hadamard), $\mathbf{T}$ (phase gate), and CNOT (controlled NOT) is a universal set of gates, meaning that every unitary can be decomposed in a circuit made of these. If any unitary can be produced, every state in the Hilbert space can be reached. Of course, also CNOT plus a random one-qubit unitary is universal. We find that evolution obtained with the full unitary group by means of universal quantum computation is irreversible and the statistics of the level spacings of reduced density matrix spectrum is Wigner-Dyson, i.e, is complex. On the other hand, if the entanglement level spacing statistics is Poisson, we find that the disentangling algorithm 
succeeds, which means that the pattern of entanglement is not complex. Strikingly, this happens when the set of gates used is not universal for quantum computing. It is remarkable that reversibility arises with severe breaking of ergodicity. If the set of gates is not universal, not every state can be reached, and ergodicity is broken.

\section{Entanglement in stochastic unitary quantum circuits}

We consider discrete quantum mechanical systems made of qubits, that is, two level systems with Hilbert space $\mathcal{H}_{i} \simeq \mathrm{C}^{2} \simeq \operatorname{span}\{|0\rangle,|1\rangle\}$, the latter being a orthonormal basis. The total Hilbert space of $n$ qubits is thus $\mathcal{H}=\otimes_{i=0}^{n-1} \mathcal{H}_{i}$, that is spanned by $2^{n}$ vectors. A basis can be conveniently written in terms of tensor product states $|k\rangle=\bigotimes_{i=0}^{n-1}\left|x_{i}\right\rangle \equiv\left|x_{n-1} \ldots x_{0}\right\rangle$ where $x_{i}=0$ or $1 . \quad k=x_{n-1} \ldots x_{0}$ is just a number expressed in binary digits; this is therefore referred to as the numerical basis. A general state of the system is a linear combination with complex numbers of these basis states, $|\Phi\rangle=\sum_{k=0}^{2^{n}-1} \phi_{k}|k\rangle$. Normalization of probability imposes that $\sum_{k=0}^{n-1}\left|\phi_{k}\right|^{2}=1$. On a classical computer, such a state can be represented by a complex vector with $2^{n}$ elements: $\left(\phi_{0}, \ldots, \phi_{2^{n}-1}\right)$.

The unitary evolution of the state is performed by applying quantum gates. Any unitary operation can be performed by a sequence of gates acting on one or two qubits at a time. Furthermore, only a finite number of such gates is needed to form the universal set of operations.

Here we consider the following sets of gates.

- NOT gate: flips the state of the qubit, $|0\rangle \rightarrow|1\rangle$ and $|1\rangle \rightarrow|0\rangle$.

- $\mathbf{H}$ (Hadamard) gate: takes $|0\rangle \rightarrow \frac{1}{\sqrt{2}}(|0\rangle+|1\rangle)$ and $|1\rangle \rightarrow \frac{1}{\sqrt{2}}(|0\rangle-|1\rangle)$.

- Phase gates $\mathbf{P}_{\delta}$ : gives a state dependent phase, $|0\rangle \rightarrow|0\rangle$ and $|1\rangle \rightarrow e^{i \delta}|1\rangle$. The phase gate with $\delta=\pi / 4$ is called $\mathbf{T}$ and that with $\delta=\pi / 2$ is called $\mathbf{S}$.

- CNOT gate or controlled-NOT gate: this is a two-qubit gate that performs a NOT on a controlled (second) bit if the control (first) bit is 1 and does nothing if the control bit is $0:|00\rangle \rightarrow|00\rangle,|01\rangle \rightarrow|01\rangle,|10\rangle \rightarrow|11\rangle,|11\rangle \rightarrow|10\rangle$.

The set $\mathcal{I}$, comprising, $\mathbf{H}, \mathbf{P}_{\delta}$, and $\mathbf{C N O T}$ is sufficient to reach arbitrarily close to any state in the Hilbert space, provided $\delta$ is such that $\theta=2 \cos ^{-1}\left(\cos ^{2}(\delta / 2)\right)$ is an irrational multiple of $\pi$, for example when $\delta=\frac{\pi}{4}$ (as in the $\mathbf{T}$ gate) [7]. The condition on $\theta$ can be understood from the requirement that the whole Bloch sphere must be accessible, and since unitary operations correspond to rotations of the Bloch sphere, this is only possible if a rotation by an irrational angle is constructable from the set of operations. In contrast, the set of gates $\mathbf{H}, \mathbf{S}$, and CNOT does not lead to universal quantum computing; this set spans a subset of operations, the Clifford group [8]. Although one can express arbitrary unitaries on the space of $n$ qubits in terms of a handful of types of gates, the number of operations needed to implement any given unitary gate may be very large, in principle countably infinite.

The inverse of any unitary operation written using a sequence of $\mathbf{H}, \mathbf{P}_{\delta}$, and CNOT gates is simply the sequence written backwards, with $\mathbf{P}_{-\delta}=\mathbf{P}_{\delta}^{-1}$ in place of 
$\mathbf{P}_{\delta}$ in the reverse sequence. That such a reverse sequence exists, with exactly the same length as the original sequence, says nothing about the difficulty of finding the reverse computation if the sequence is concealed.

The statement of universality of a set of gates $\mathcal{I}$ means that any unitary operator $U \in \mathcal{U}(\mathcal{H})$ can be well approximated as the product of $U_{a} \in \mathcal{I}$, that is, $U \simeq \prod_{a=1}^{K} U_{a}$. The unitary $U$ is called quantum circuit. In the following, we consider stochastic quantum circuits. A stochastic quantum circuit of $K$ gates is obtained by randomly selecting $K$ gates $U_{a} \in \mathcal{I}$, as well as the either one or two qubits on which such gates act.

We are interested in studying several entanglement properties of states that are obtained by evolving with a stochastic quantum circuit $U$. The initial state is a pure state $|\Psi\rangle$ with the completely factorized form

$$
\left|\Psi_{0}\right\rangle=\left|\psi_{0}\right\rangle \otimes\left|\psi_{1}\right\rangle \otimes \cdots\left|\psi_{n-1}\right\rangle
$$

where

$$
\left|\psi_{j}\right\rangle=\cos \left(\theta_{j}\right)|0\rangle_{j}+\sin \left(\theta_{j}\right)|1\rangle_{j}
$$

Being $\left|\Psi_{0}\right\rangle$ completely factorized, every restriction of the state to any subsystem $A$ is still a pure state. On the other hand, if $\left|\Psi_{0}\right\rangle$ were entangled, we would obtain a classical probability distribution for a mixed state. Entanglement is relative to the partitioning of the system in two parts $A, B$, that is, by considering the Hilbert spaces $\mathcal{H}_{A}, \mathcal{H}_{B}$ of $n_{A}, n_{B}$ given qubits such that $\mathcal{H}=\mathcal{H}_{A} \otimes \mathcal{H}_{B}$, where $n=n_{A}+n_{B}$. Given a (pure) state $|\psi\rangle \in \mathcal{H}$, the reduced density matrix $\rho_{A}$ associated to the restricted system $A$ is obtained by tracing out the qubits in $B$ :

$$
\rho_{A}=\operatorname{tr}_{B}|\psi\rangle\langle\psi|
$$

The eigenvalues of $\rho_{A}$, that is, $\operatorname{spec}\left\{\rho_{A}\right\}=\left\{\lambda_{1}, \ldots, \lambda_{2}{ }^{n_{A}}\right\}$, form the reduced density matrix spectrum. They are indeed just the spectrum of the Hermitian operator $\rho_{A}$. The operator $\rho_{A}$, being a density matrix, has unit trace, so $\sum_{i=1}^{2^{n} A} \lambda_{i}=1$, with $\lambda_{i} \geq 0$, thus representing a classical probability distribution. In the following, we are interested in studying the spectrum of the reduced states $\rho_{A}^{U}$ after the quantum circuit $U$ has been applied on the initial fiducial state $\left|\Psi_{0}\right\rangle$ :

$$
\rho_{A}^{U}=\operatorname{tr}_{B} \rho^{U}=\operatorname{tr}_{B}\left(U\left|\Psi_{0}\right\rangle\left\langle\Psi_{0}\right| U^{\dagger}\right)
$$

The entropy measures of the probability distribution $\left\{\lambda_{i}\right\}$ associated to $\rho_{A}^{U}$ are given by the Rényi entropies $S_{\alpha}$, namely

$$
S_{q}\left(\rho_{A}^{U}\right)=\frac{1}{1-q} \log _{2} \operatorname{tr}\left(\rho_{A}^{U}\right)^{q}
$$

In the limit $q=1$, this is the von Neumann entropy of entanglement, while $S_{0}$ is the number of nonzero eigenvalues of $\rho_{A}^{U}$, i.e., its rank. Of course, for a factorized state all Rényi entropies $S_{q}=0$ for all $q$. Moreover, we recall the useful inequality $S_{q} \geq S_{q^{\prime}}$ for $q<q^{\prime}$. Notice that the Rényi entropies depend explicitly on the choice of the subsystem $A$ and on the circuit $U$. So, for every circuit $U$ and every partition $A$, we obtain a set of 
quantities $S_{\alpha}\left(\rho_{A}^{U}\right)$. As we mentioned above, the stochastic quantum circuit $U$ is made of $K$ gates, so we may denote it by $U(K)$. Here, $K$ plays the role of time. At every click of the clock, we apply a new gate and make the circuit longer. Given a number $K$ and a partition $A$, one can make many realizations of the quantum circuit $U(K)$. Then, it makes sense to consider the averages of the Rényi entropies over the many circuit realizations, namely $S(q, K, A) \equiv{\overline{S_{q}\left(\rho_{A}^{U}(K)\right)}}^{U}$. Again, this quantity depends explicitly on $K$. As $K$ increases, one expects $\bar{S}_{q}\left(\rho_{A}^{U}(K)\right)^{U}$ to start at zero and monotonically increase to the maximum possible value of the entropy, as it was shown in Ref. [9]. After we reach a state with maximum entanglement for a given system size $n$, we can try to disentangle it without resorting to the inverse of circuit $U(K)$, namely, $U(K)^{\dagger}$. Of course, by exhaustive search, one can try all possible quantum circuits for a given set of gates until we find the right one. This search is very expensive and, already for some dozens of qubits, it is prohibitive. If there is nothing better than brute search, the entanglement is too complex to be undone, and we say that the evolution is irreversible. On the other hand, if there is an efficient way of finding a circuit $U\left(K^{\prime}\right)$ such that $U\left(K^{\prime}\right) U(K)\left|\Psi_{0}\right\rangle$ is completely factorized, then the entanglement is not complex, and the evolution is reversible. The algorithm used to find the disentangling algorithm resembles the Metropolis algorithm to reach the ground state of a quantum system, and we thus dub it entanglement cooling. The algorithm will be explained in the next section.

At this point, it is desirable to characterize the entanglement in the state $U(K)\left|\Psi_{0}\right\rangle$ without having to try to disentangle it. Is there anything in the entanglement itself that shows us, beforehand, whether the entanglement cooling will work or not? As shown in Ref. [6], the answer is hidden in the statistics of the level spacing for the entanglement spectrum. To be precise, we order the members of $\operatorname{spec}\left(\rho_{A}^{U}\right)$ in decreasing order, and consider the distribution of the ratio of consecutive spacings, namely, $P(r)=\frac{1}{R} \sum_{i=1}^{R}\left\langle\delta\left(r-r_{i}\right)\right\rangle$, where $r_{i}=\epsilon_{i+1} / \epsilon_{i}$ and $\left\{\epsilon_{i}=\lambda_{i}-\lambda_{i+1}\right\}_{i=1, \ldots, R}$. As we shall show, irreversibility is associated to a Wigner-Dyson statistics for this distribution. Moreover, if $U$ is obtained by $\mathcal{I}$, this is always the case. On the other hand, if the quantum circuits are not drawn from a universal set, the entanglement cooling algorithm always works, entanglement is not complex, and the distribution $P(r)$ deviates from Wigner-Dyson prediction and is akin to a Poisson one. This is the main result of the paper.

\section{Simulation Setup}

In this section, we describe the detail of the simulations and results. Our system comprises 16 qubits. The simulations are made of three parts: entanglement heating, entanglement cooling, and level spacing statistics. 


\subsection{Entanglement heating}

We refer to the initial evolution by the stochastic random circuit $U$ as entanglement heating, since, as we shall see, we start from a completely factorized state until reaching maximum entanglement, as one would expect from a random quantum circuit [9]. The circuit $U$ is obtained by drawing random gates from different sets. The set $\mathcal{I}$, as mentioned above, is universal and comprises $\mathbf{H}, \mathbf{T}$, and CNOT. The sets used are therefore

$$
\begin{aligned}
\mathcal{I} & =\{C N O T+H+T\} \\
\text { or } \quad \mathcal{I} & =\{C N O T+N O T+H\} \\
\text { or } \quad \mathcal{I} & =\{C N O T+H+S\}
\end{aligned}
$$

The initial state $\left|\Psi_{0}\right\rangle$ is always a (random) factorized as explained in the previous section. The random state is chosen by randomizing the $\theta_{j}$ in Eq.(2) with uniform probability over the interval $[0: \pi]$. In the simulation, we first randomly select a qubit, then with uniform probability choose what gate to apply (and, in the case when a two-qubit gate is picked, the second qubit is then randomly chosen as well). The heating circuit $U$ is made of 512 gates. From the graph theoretic point of view, we have a random quantum circuit on a complete graph. All heating simulations are conducted with 5000 realizations. For every realization of $U$, we compute $S_{0}\left(\rho_{A}^{U}\right), S_{1}\left(\rho_{A}^{U}\right)$ where $A$ is a subset with 8 qubits. Then we average over all the realizations, generating ${\overline{S_{0}\left(\rho_{A}^{U}\right)}}^{U}$ and ${\overline{S_{1}\left(\rho_{A}^{U}\right)}}^{U}$. In Fig. 1 we show the average Rényi entropies as a function of the number of gates applied for the three circuit cases. For this plot, the entropy was obtained after bipartitioning the bit string into two equal parts, with $A$ corresponding to bits 0 to 7 and $B$ corresponding to bits 8 to 15 . Notice that while ${\overline{S_{0}}}^{U}$ always reach its maximum possible value after a few hundred gates, the saturation value of ${\overline{S_{1}}}^{U}$ varies from circuit to circuit and is not a good indication of whether the circuit is reversible or not by the cooling algorithm. The maximum saturation value is expected to happen in the thermodynamic limit. We clearly see that the different sets of gates are not distinguished at all by the way entanglement increases and saturates. In particular, entanglement is not able to tell apart universal from non-universal set of gates.

\subsection{Entanglement cooling and level spacing statistics}

In our simulation, we start with a random product state, so the entanglement entropy increases from zero to a maximum value after a certain number $\left(\sim n^{2}\right)$ of gates. We therefore refer to this part of the process as entanglement heating. After a state reaches the maximum entanglement observed for that system size, our task is thus to disentangle

it, i.e., reduce the entanglement entropy to zero without using knowledge of the random circuit. As alluded to above, the procedure is a Metropolis-like algorithm: we apply a gate on the entangled state at random, chosen from the same set of gates from which the random circuit was constructed; if the entanglement entropy decreases, we keep the gate; if it increases, we reject the gate. The process goes on until the entanglement entropy is 

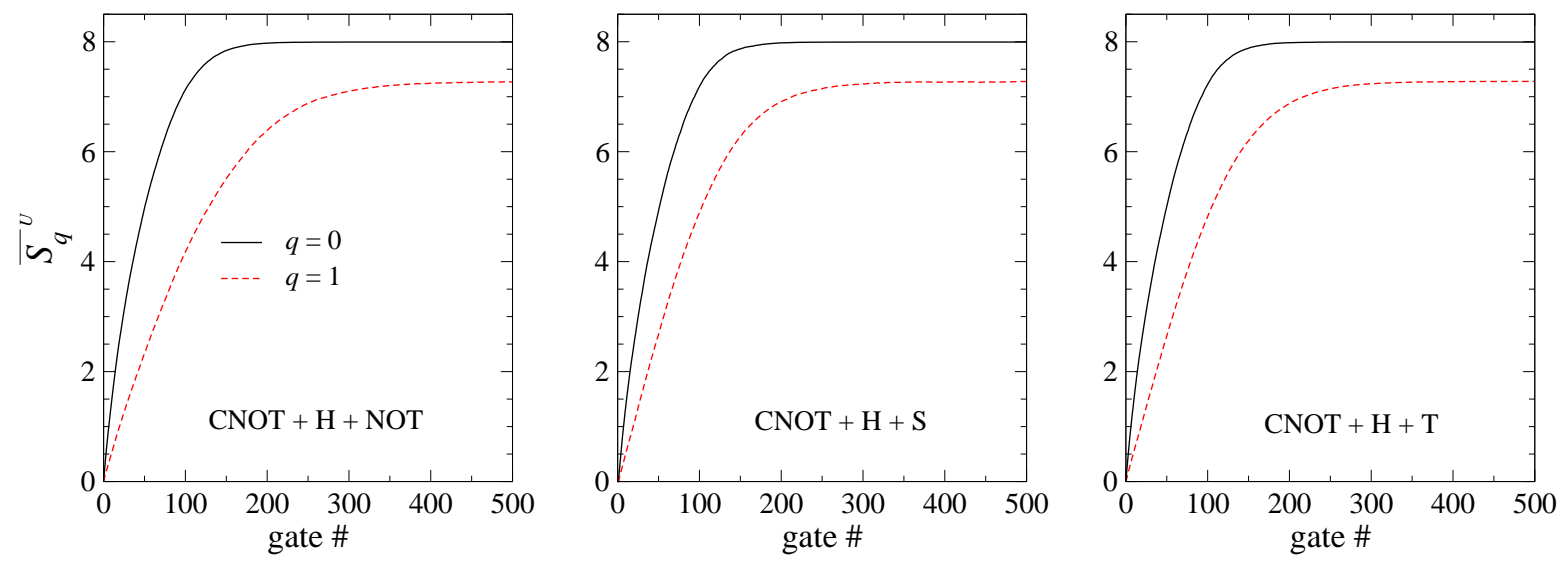

Figure 1. Average entanglement entropies ${\overline{S_{0}}}^{U}$ (solid line) and ${\overline{S_{1}}}^{U}$ (dashed line) as a function of gate number for three circuit cases. The cases CNOT+H+NOT and $\mathbf{C N O T}+\mathbf{H}+\mathbf{S}$ are reversible, while the case $\mathbf{C N O T}+\mathbf{H}+\mathbf{T}$ is not. Notice that the entanglement entropies themselves do not flag which circuit is easily reversed or not. The complexity of the entanglement is instead contained in the reduced density matrix spectrum level statistics presented below.

zero, or until we give up. In order to reach the completely factorized state, we actually consider the average of particular bipartitions $(A, B)$ of the system with $n_{A}=n-n_{B}$ running from 1 to $n-1$. These bipartitions are constructed by laying down the qubits on a line, and taking the $n-1$ possible cuts; system $A$ is always chosen to be the qubits on the left, and $B$ those on the right. Also, more generally than simply rejecting the gates that increase the entanglement, we actually use a simulated annealing type variant, where we accept the increase in entropy with a probability $\exp \left[-\beta\left(S_{\text {new }}-S_{\text {old }}\right)\right]$, with $\beta$ chosen as $\beta=5$ in units of entropy. We refer to this part of the simulation as entanglement cooling. The algorithm is summarized below.

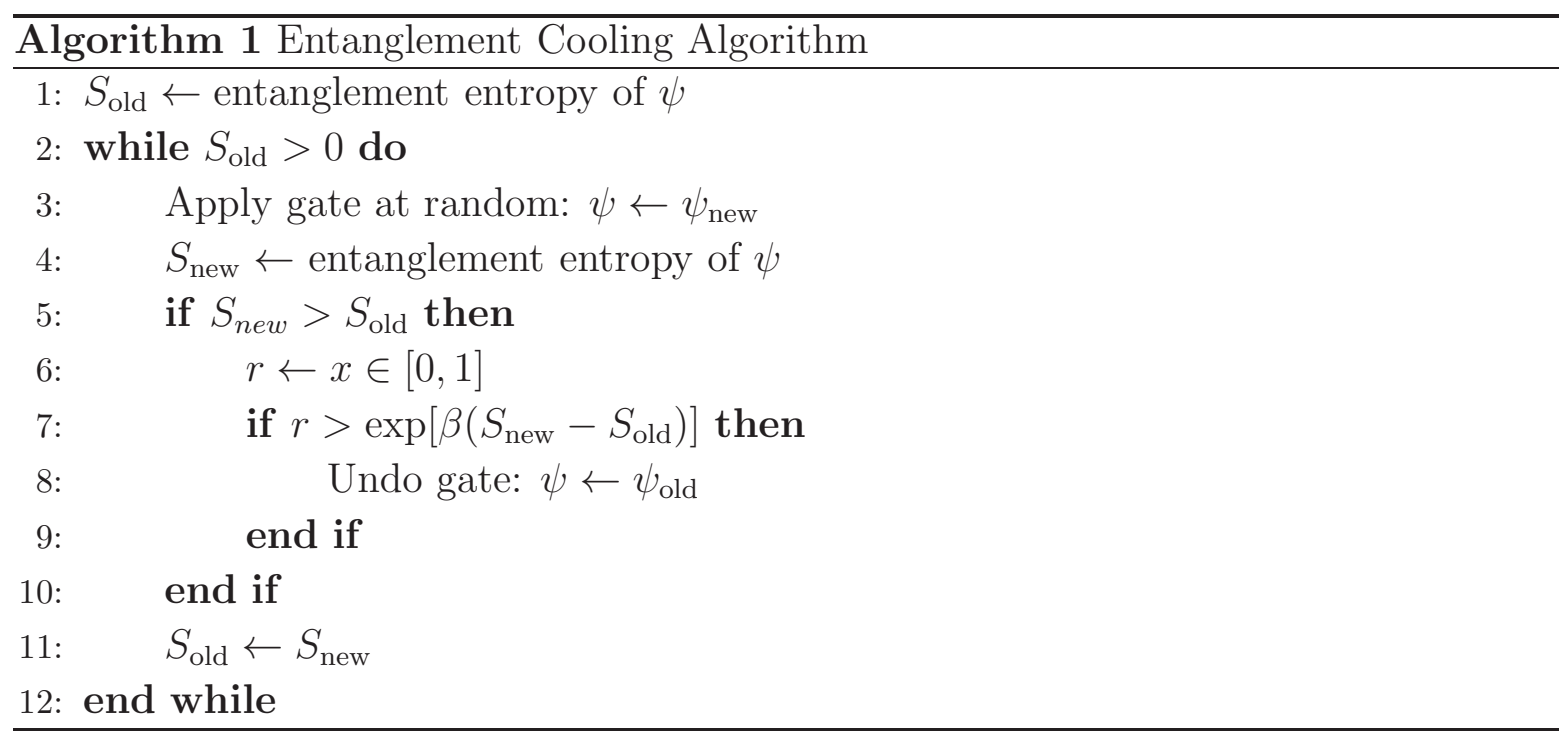

The cooling algorithm is computationally heavy on a classical machine because 
it involves a singular value decomposition. Furthermore, it is necessary at each step to compute not just a single entanglement entropy, but entanglement entropies over all possible ordered bipartitions. This is due to the fact that even if the state is a product of two substates, the two substates may themselves be entangled. On a quantum machine, the algorithm could be accelerated by replacing the entanglement entropy with the second Rényi entropy, which is an observable quantity and so could be used during computation [6]. Such performance boosts of classical algorithms is the main thrust behind the development of a quantum computer.

Not having a quantum machine, however, we may want to know whether the algorithm will fail before we attempt it. In that case, we would probably not want to run it on a quantum machine either. The point of the proposed conjecture is that it does not require any computation to be performed on the state; the state itself is all that is needed to obtain the reduced density matrix spectrum. After heating up the state, and before we attempt to cool it, we therefore look at its entanglement spectrum. Because the heating process is fast, we can obtain spectra over many realizations of the system. If we observe level repulsion, i.e., a Wigner-Dyson level spacing behavior, we predict that the system will not be reversed by the cooling algorithm.

In practice, evaluating the level spacing distribution directly is difficult because of the enormous variations in the level density. In Ref. [6], an elaborate unfolding procedure was used to make the reduced density matrix spectrum uniform. Here, to avoid this procedure, we evaluate the distribution of ratios of adjacent level spacings. An analysis of spectral fluctuations based on such distribution was first used (for the case of energy, but not entanglement levels) in studies of finite-temperature manybody localization by Oganesyan and Huse [10]. Later, very accurate surmises for this distribution were derived by Atas and coworkers [11] for the Gaussian ensembles. They are given by the expression

$$
P_{\mathrm{WD}}(r)=\frac{1}{Z} \frac{\left(r+r^{2}\right)^{\beta}}{\left(1+r+r^{2}\right)^{1+3 \beta / 2}},
$$

where $Z=\frac{8}{27}$ for the Gaussian Orthogonal Ensemble (GOE) with $\beta=1$, and $Z=\frac{4}{81} \frac{\pi}{\sqrt{3}}$ for the Gaussian Unitary Ensemble (GUE) with $\beta=2$. The corresponding distribution for a spectrum with Poisson statistics is

$$
P_{\text {Poisson }}(r)=\frac{1}{(1+r)^{2}} \text {. }
$$

Notice that $P_{\mathrm{WD}}(r \rightarrow 0) \sim r^{\beta} \rightarrow 0$, which indicates level repulsion, while $P_{\text {Poisson }}(r \rightarrow$ $0) \rightarrow 1$. The tails of these distributions are also markedly different, with $P_{\mathrm{WD}}(r \rightarrow$ $\infty) \sim 1 / r^{2+\beta}$ while $P_{\text {Poisson }}(r \rightarrow \infty) \sim 1 / r^{2}$.

For the "cooling" simulations, out of the 5000 samples, we picked randomly 100 samples and attempted to use the disentangling algorithm to return their maximally entangled states back to a product state form. The restricted number is due to the fact that the cooling algorithm is very time consuming. The results of the simulation 
are presented below. However, for the statistical analysis, we used all 5000 samples generated for each circuit set.

For each circuit case, we show a typical evolution of the entanglement entropy as gates are applied, both during the "heating" and "cooling" processes. Other plots show the resulting eigenvalue level spacing ratio distribution collected at the end of the heating process. To highlight the behavior of the distributions at the tails, we show the data in both linear and logarithm scales.

3.2.1. CNOT $+\mathbf{H}+\mathbf{N O T}$ This ensemble of gates is not universal. For all 100 samples tested, the disentangling algorithm was able to brought the state back to a direct product form. See, for example, the run shown in Fig. 2(a). The spacing ratio distribution follows closely a Poisson curve, as shown in Figs. 2(b,c).

3.2.2. $\mathbf{C N O T}+\mathbf{H}+\mathbf{S}$ As in the previous case, this set is not universal [8]. The cooling algorithm is able to systematically reduce both $S_{0}$ and $S_{1}$ entropies with no tendency for saturation, although sometimes we were not able to reach the zero value within the maximum number of steps used (200000). In Fig. 3 we show a case where nearly a complete disentanglement was obtained. The distribution of spacing ratios follows closely a Poisson curve, as indicated in Figs. 3(b,c). We can therefore establish that Poisson statistics correspond to reversibility of the stochastic quantum evolution.

3.2.3. $\mathbf{C N O T}+\mathbf{H}+\mathbf{T}$ This is the universal set of gates $\mathcal{I}$. We find that for all 100 samples tested, the disentangling algorithm was not only unable to brought the state back to a direct product, but not even to disentangle the state partially, as can be seen in the instance shown in Fig. 4(a). Notice that in this case neither $S_{0}$ nor $S_{1}$ are reduced by the cooling algorithm. Figures $4(\mathrm{~b}, \mathrm{c})$ show that the distribution of spacing ratios in this case follows the GUE prediction.

\section{Conclusions and outlook}

In this paper, we have shown that irreversibility and chaotic behavior in quantum mechanics corresponds to a complex pattern of entanglement that cannot be undone by entanglement cooling. This pattern is revealed by Wigner-Dyson statistics in the entanglement level spacing statistics. We showed that universal quantum computation always produces complex entanglement. Universal quantum computation means that every state in the Hilbert space can be reached, so complexity of entanglement and irreversibility correspond to ergodicity. On the other hand, non-universal quantum computation results in a breaking of ergodicity and this is revealed in Poisson level spacing statistics, in turn ensuring that entanglement is not complex and easily undone.

The results are summarized in the table below. Universal computation is always irreversible: 

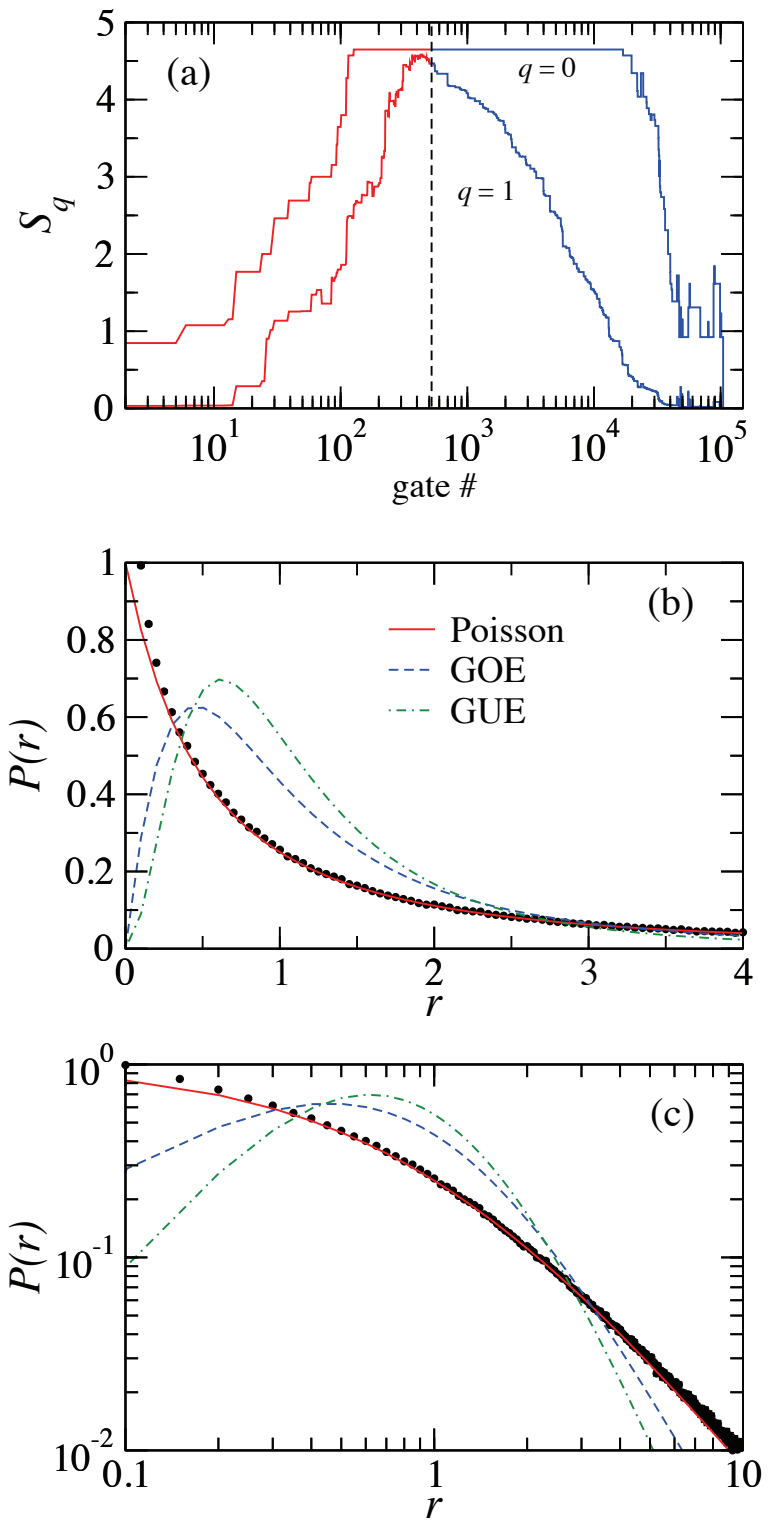

Figure 2. CNOT $+\mathrm{H}+\mathrm{NOT}$ circuit. (a) Sample evolution of the entanglement entropies $S_{0}$ and $S_{1}$ (averaged over $n-1$ bipartitions) as functions of gate number. The dashed line separates the heating and cooling processes (notice that the gate number is given in logarithmic scale). (b) and (c): distribution of eigenvalue spacing ratios in linear and logarithmic scales, respectively. The solid circles represent the numerical data while the solid, dashed, and the dotted lines are the Poisson, GOE, and GUE predictions, respectively.

\begin{tabular}{|l|c|c|}
\hline Operator Set & $\begin{array}{l}\text { Level } \\
\text { Spacing } \\
\text { Statistics }\end{array}$ & Reversible? \\
\hline$\{C N O T, H, N O T\}$ & Poisson & Yes \\
\hline$\{C N O T, H, S\}$ & Poisson & Yes \\
\hline$\{C N O T, H, T\}$ & GUE & No \\
\hline
\end{tabular}



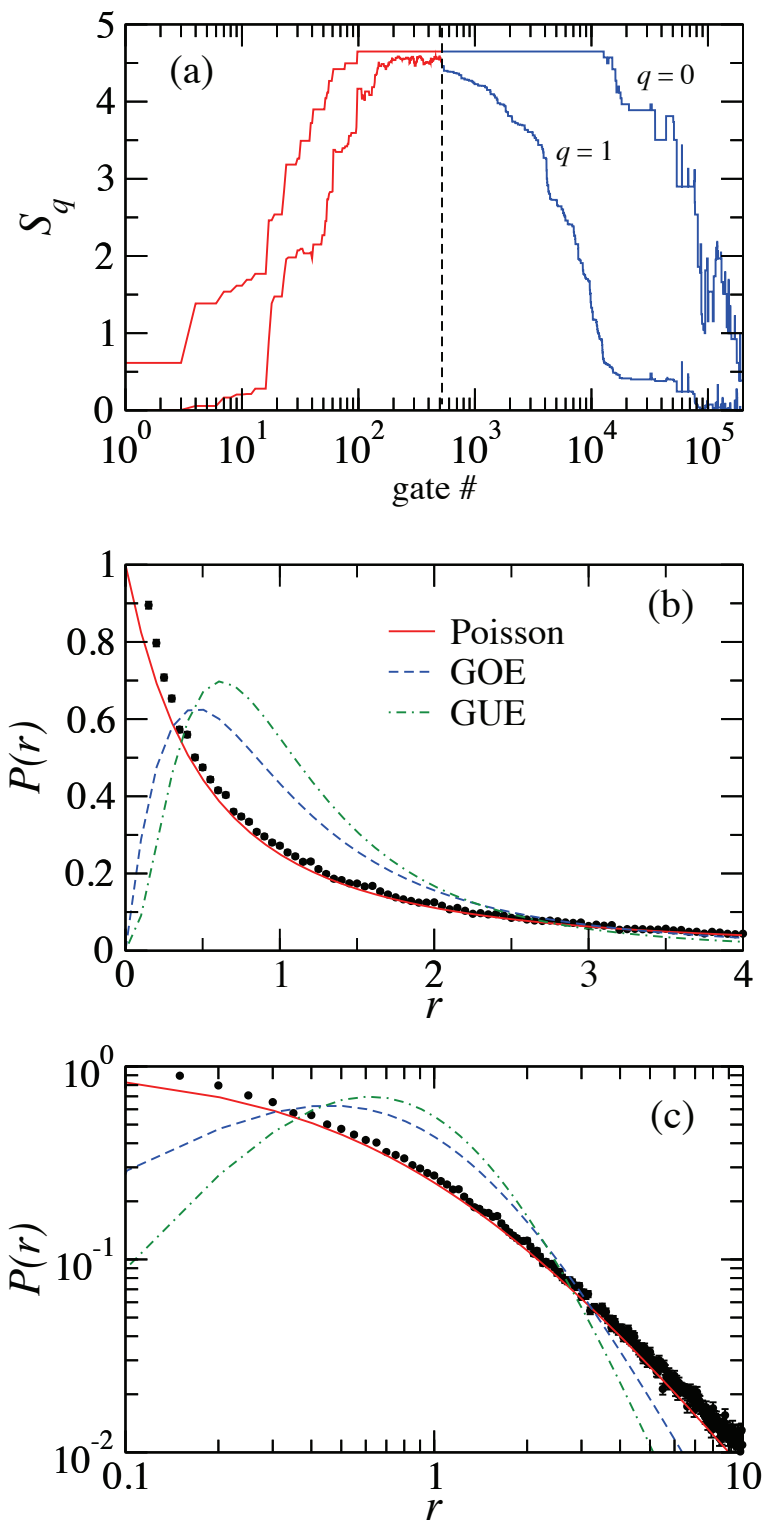

Figure 3. CNOT $+\mathrm{H}+\mathrm{S}$ circuit. (a) Sample evolution of the entanglement entropies as functions of gate number. (b) and (c): Distribution of eigenvalue spacing ratios (see caption of Fig. 21).

It is important to emphasize that it is not the amount of entanglement in itself that counts. Typically (that is, it is almost always the case) quantum evolution brings about the maximum possible entanglement. But just as some knots are easy to undo and some are not, this happens to entanglement as well. The complexity of entanglement is revealed by the level spacing statistics. We speculate that signatures of this behavior may be found in higher moments of the entanglement entropy, since after all, knowledge of all the Rényi entropy should in principle allow to reconstruct the whole reduced density matrix spectrum.

Another point that is worth emphasizing is that this way of looking at quantum 

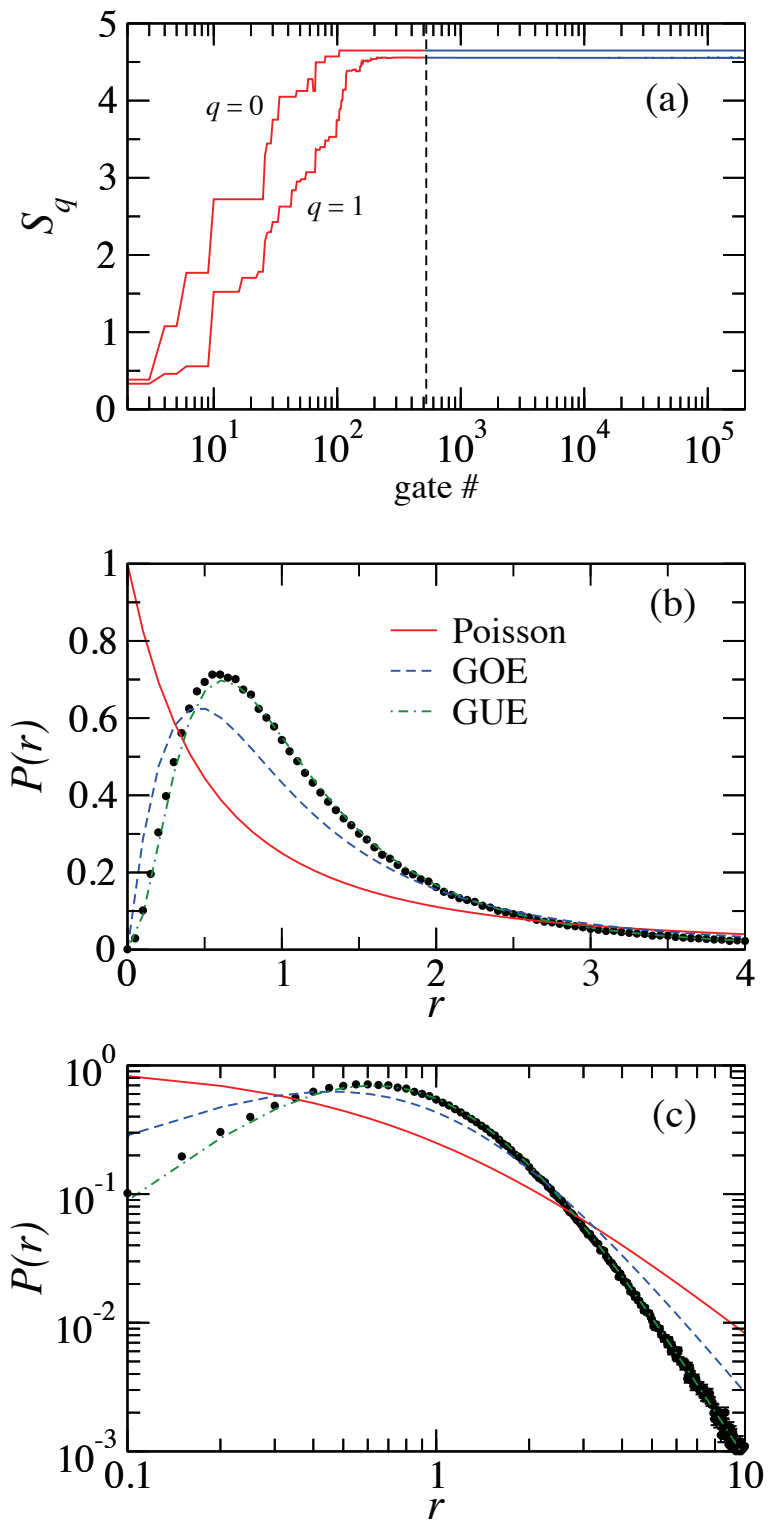

Figure 4. $\mathrm{CNOT}+\mathrm{H}+\mathrm{T}$ circuit. (a) Sample evolution of the entanglement entropies as functions of gate number. (b) and (c): Distribution of eigenvalue spacing ratios (see caption of Fig. 2).

evolutions is very general. We do not need a Hamiltonian. This means that generic quantum evolutions (even non-unitary, like in open quantum systems) can be explored in the same way. All we need is the density matrix.

The results in this work open a way to characterize, through entanglement, quantum systems that do not thermalize, as breaking of thermalization must come from breaking of ergodicity. Therefore, long lived quantum memories or quantum states that feature many-body localization, should feature deviations from Wigner-Dyson statistics in their entanglement level spacing statistics. We believe that this study can shed new light on questions regarding quantum many-body physics away from equilibrium, and help clarify 
the notions of thermalization and irreversibility.

\section{Acknowledgments}

This work was supported in part by the NSF grants CCF-1116590 and CCF1117241, and by the National Basic Research Program of China Grant 2011CBA00300, 2011CBA00301, the National Natural Science Foundation of China Grant 61033001, 61361136003.

\section{References}

[1] Ter Haar D, Foundations of Statistical Mechanics, 1955 Rev. Mod. Phys. 27 p 289

[2] Popescu S, Short A J and Winter A, Entanglement and the foundations of statistical mechanics, 2006 Nat. Phys. 2 754-758

[3] A. Peres, Stability of quantum motion in chaotic and regular systems, 1984 Phys. Rev. A 301610

[4] Benenti G and Casati G, How complex is quantum motion, 2009 Phys. Rev. E 79 025201(R)

[5] Zanardi P, Lidar D and Lloyd S, Quantum tensor product structures are observable induced, 2004 Phys. Rev. Lett. 92060402

[6] Chamon C, Hamma A and Mucciolo E R, Emergent irreversibility and entanglement spectrum statistics, 2014 Phys. Rev. Lett. 112240501

[7] Nielsen M A and Chuang I L 2001, Quantum Computation and Quantum Information (Cambridge University Press) ch. 4

[8] Gottesman D, The Heisenberg representation of quantum computers, 1999 in Proc. XXII International Colloquium on Group Theoretical Methods in Physics (Cambridge: International Press) pp 32-43

[9] Hamma A, Santra S and Zanardi P, Quantum entanglement in random physical states, 2012 Phys. Rev. Lett. 109040502

[10] Oganesyan V and Huse D A, Localization of interacting fermions at high temperature, 2007 Phys. Rev. B 75155111

[11] Atas Y Y, Bogomolny E, Giraud O and Roux G, Distribution of the ratio of consecutive level spacings in random matrix ensembles, 2013 Phys. Rev. Lett. 110084101 